\title{
Self-Diffusion in Amorphous Silicon by Local Bond Rearrangements
}

\author{
J. Kirschbaum, ${ }^{1}$ T. Teuber, ${ }^{1}$ A. Donner, ${ }^{1}$ M. Radek, ${ }^{1}$ D. Bougeard, ${ }^{2}$ R. Böttger,${ }^{3}$ J. Lundsgaard Hansen, ${ }^{4}$ \\ A. Nylandsted Larsen, ${ }^{4}$ M. Posselt, ${ }^{3, *}$ and H. Bracht ${ }^{1, \dagger}$ \\ ${ }^{1}$ Institute of Materials Physics, Westfälische Wilhelms-Universität Münster, 48149 Münster, Germany \\ ${ }^{2}$ Institut für Experimentelle und Angewandte Physik, Universität Regensburg, 93040 Regensburg, Germany \\ ${ }^{3}$ Helmholtz-Zentrum Dresden-Rossendorf, 01328 Dresden, Germany \\ ${ }^{4}$ Department of Physics and Astronomy, Aarhus University, 8000 Aarhus, Denmark
}

(Received 9 March 2018; published 31 May 2018)

\begin{abstract}
Experiments on self-diffusion in amorphous silicon ( $\mathrm{Si}$ ) were performed at temperatures between 460 to $600^{\circ} \mathrm{C}$. The amorphous structure was prepared by $\mathrm{Si}$ ion implantation of single crystalline Si isotope multilayers epitaxially grown on a silicon-on-insulator wafer. The Si isotope profiles before and after annealing were determined by means of secondary ion mass spectrometry. Isothermal diffusion experiments reveal that structural relaxation does not cause any significant intermixing of the isotope interfaces whereas self-diffusion is significant before the structure recrystallizes. The temperature dependence of selfdiffusion is described by an Arrhenius law with an activation enthalpy $Q=(2.70 \pm 0.11) \mathrm{eV}$ and preexponential factor $D_{0}=\left(5.5_{-3.7}^{+11.1}\right) \times 10^{-2} \mathrm{~cm}^{2} \mathrm{~s}^{-1}$. Remarkably, $Q$ equals the activation enthalpy of hydrogen diffusion in amorphous $\mathrm{Si}$, the migration of bond defects determining boron diffusion, and the activation enthalpy of solid phase epitaxial recrystallization reported in the literature. This close agreement provides strong evidence that self-diffusion is mediated by local bond rearrangements rather than by the migration of extended defects as suggested by Strauß et al. (Phys. Rev. Lett. 116, 025901 (2016)).
\end{abstract}

DOI: 10.1103/PhysRevLett.120.225902

Self-diffusion in single-crystalline silicon $(c-\mathrm{Si})$ is a well-known phenomenon that was extensively studied in the past. Self-diffusion in $c$-Si is mediated by both vacancies and self-interstitials [1,2]. In contrast, first experimental results on self-diffusion in amorphous $\mathrm{Si}$ ( $a$-Si) have been published only recently $[3,4]$. The problem in obtaining the self-diffusion coefficient for $a$ $\mathrm{Si}$ is due to the difficulty to suppress two processes competing with self-diffusion: (i) the random nucleation of the crystalline phase and (ii) the solid phase epitaxial recrystallization (SPER) of the $a$-Si layer commonly prepared on respective $c$-Si substrates. In the studies of Strauß et al. $[3,4]$, an amorphous ${ }^{29} \mathrm{Si} /{ }^{\text {nat }} \mathrm{Si}$ isotope structure was deposited directly on a $c$-Si wafer using ion-beam sputtering. No detailed information on the pre-treatment of the single crystalline substrate wafer before layer deposition and on the thickness of its native oxide before and after sputter deposition is reported. This information is highly relevant to judge whether SPER is to be expected during the diffusion anneals performed by Strauß et al. $[3,4]$. In this context it is also noted that a very thin oxide layer between substrate wafer and $a$-Si not necessarily prevents SPER but even can mediate the growth of a single crystalline layer [5]. Considering the SPER velocity reported in the literature $[6,7]$, the amorphous layer prepared by Strauß et al. should be fully recrystallized during the diffusion anneals performed in their work. Taking into account the activation enthalpy of $Q=2.75 \mathrm{eV}$ and prefactor $v_{0}=3.68 \times 10^{8} \mathrm{~cm} / \mathrm{s}$ for the SPER velocity given by Ref. [6], the expected SPER crystallization time at $550^{\circ} \mathrm{C}\left(600^{\circ} \mathrm{C}\right)$ for a $310 \mathrm{~nm}$ thick $a$-Si, which equals the layer thickness prepared by Strauß et al., is $5788 \mathrm{~s}$ (629 s). Strauß et al. [3,4] report significantly longer diffusion times of $254520 \mathrm{~s}(14400 \mathrm{~s})$ for annealing at $550^{\circ} \mathrm{C}\left(600^{\circ} \mathrm{C}\right)$. Unfortunately, they did not discuss whether the native oxide layer between the $a$-Si layer and the $c$-Si substrate and/or the high level of carbon contamination (5\% at maximum) in their amorphous ${ }^{29} \mathrm{Si} /{ }^{\text {nat }} \mathrm{Si}$ isotope multilayers (see supplemental material of [3] and Ref. [4]) have suppressed SPER. In particular, carbon impurities can lead to a significant retardation of SPER [8,9]. Thus it is likely that SPER in the samples of Strauß is effectively suppressed by the high level of carbon impurities in the amorphous isotope structure rather than by a native oxide between the amorphous layer and the crystalline $\mathrm{Si}$ substrate. Overall, the missing discussion on SPER and on the impact of carbon impurities in the work of Strauß et al. $[3,4]$ questions their self-diffusion data to be representative for high purity $a-\mathrm{Si}$.

Several methods are reported in the literature to prepare homogeneous and compact $a$-Si layers. The most favoured method is amorphization of $c$-Si by ion implantation (cf. e.g. Refs. [6,7,10,11]), ion-beam or plasma sputter deposition (cf. e.g. Refs. [3,4,12]), and laser melting followed by rapid quenching (cf. e.g. Ref. [10]). These procedures ensure the formation of $a$-Si without voids and 
inhomogeneities. As-prepared $a$-Si samples generally reveal a structural relaxation during thermal treatments as demonstrated by calorimetric measurements [10,12-15]. It is therefore important to separate the relatively fast process of structural relaxation from the long-term selfdiffusion. Due to the different time-scale of these processes the impact of relaxation and self-diffusion on the interfacial broadening of the isotope structure can be separated when isothermal diffusion experiments are preformed for various times.

In order to study self-diffusion in $a$-Si, ten bilayers of ${ }^{\text {nat }} \mathrm{Si}(10 \mathrm{~nm}) /{ }^{28} \mathrm{Si}(15 \mathrm{~nm})$ were first epitaxially grown on top of a silicon-on-insulator (SOI) wafer by means of molecular beam epitaxy (MBE). Subsequently, the top single crystalline Si layer was amorphized by twofold (sample set \#1) and threefold (sample set \#2) Si ion implantation. Details on the SOI wafer, the implantation parameters for amorphization, the microscopic characterization of the $a-\mathrm{Si}$, the diffusion experiments, and the analyses of $\mathrm{Si}$ diffusion profiles by means of Time-of-flight (TOF) secondary ion mass spectrometry (SIMS) are given in the Supplemental Material [16]. SIMS analysis of $a$-Si structure \#2 yields a carbon concentration of about $1.8 \times 10^{18} \mathrm{~cm}^{-3}$ and an oxygen concentration below the detection limit of $1.0 \times 10^{18} \mathrm{~cm}^{-3}$ (see Supplemental Material [16]).

Amorphous Si prepared by self-ion implantation undergoes a structural relaxation upon annealing that has been characterized by differential scanning and isothermal calorimetry by Roorda et al. [10]. For temperatures $\leq 500^{\circ} \mathrm{C}$ the calorimetric signal has stabilized after 10 min [10] indicating a significant relaxation process within the first minutes of annealing. To demonstrate the impact of relaxation on the interfacial broadening, Fig. 1 shows

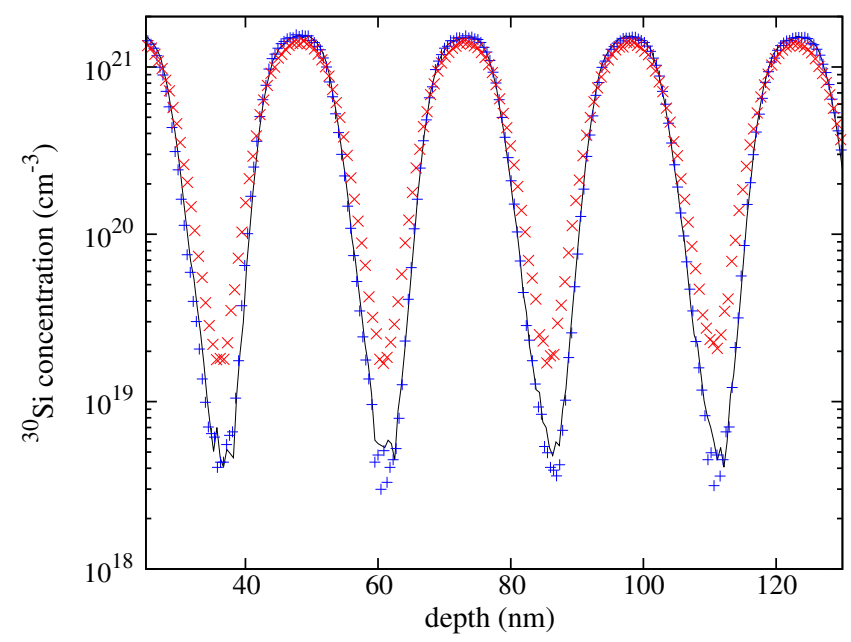

FIG. 1. SIMS concentration-depth profiles of ${ }^{30} \mathrm{Si}$ of samples from type \#2. The black solid line, blue symbols $(+)$, and red symbols $(\times)$ represent ${ }^{30} \mathrm{Si}$ profiles after amorphization, relaxation $\left(T=520^{\circ} \mathrm{C}, t=15 \mathrm{~min}\right)$, and annealing for prolonged times ( $\left.T=520^{\circ} \mathrm{C}, t=12 \mathrm{~h}\right)$, respectively.
${ }^{30} \mathrm{Si}$ concentration profiles of an amorphized sample in comparison to those of samples relaxed and annealed at $520^{\circ} \mathrm{C}$ for $15 \mathrm{~min}$ and $12 \mathrm{~h}$, respectively. Analyses of the thermal broadening based on Eq. (S1) of the Supplemental Material [16] yields $\bar{r}=(2.9 \pm 0.5) \mathrm{nm}$ for $15 \mathrm{~min}$ and $\bar{r}=(4.0 \pm 0.3) \mathrm{nm}$ for $12 \mathrm{~h}$ annealing at $520^{\circ} \mathrm{C}$. Within the depth resolution of SIMS, the broadening $\bar{r}=2.9 \mathrm{~nm}$ obtained after 15 min equals the broadening $\bar{r}=2.8 \mathrm{~nm}$ of the amorphized sample \#2. Obviously, structural relaxation is a local and short-range ordering process as reported by Roorda et al. [10] and thus does not significantly affect the broadening at the $\mathrm{Si}$ isotope interfaces. This implies that any interfacial broadening determined with SIMS after annealing is due to self-diffusion in the amorphous state.

After SIMS profiling the self-diffusion coefficient $D_{\mathrm{Si}}^{\mathrm{SD}}$ is obtained by fitting the numerical solution of Fick's second law of self-diffusion

$$
\frac{\partial C_{\mathrm{Si}}}{\partial t}-D_{\mathrm{Si}}^{\mathrm{SD}} \frac{\partial^{2} C_{\mathrm{Si}}}{\partial x^{2}}=0
$$

to the experimental ${ }^{30} \mathrm{Si}$ profiles. The profile measured after relaxation is considered as initial profile. Fig. 2 illustrates the interfacial broadening of the amorphous structure \#1 after diffusion annealing at $550^{\circ} \mathrm{C}$ for $5 \mathrm{~h}$ in comparison to the relaxed state represented by the ${ }^{30} \mathrm{Si}$ profile after annealing at $520^{\circ} \mathrm{C}$ for $15 \mathrm{~min}$. A pronounced difference in the interfacial broadening is evident. Analysis of the diffusional broadening yields $D_{\mathrm{Si}}^{\mathrm{SD}}\left(550^{\circ} \mathrm{C}\right)=(1.8 \pm 0.4) \times 10^{-18} \mathrm{~cm}^{2} / \mathrm{s}$. The solid line in Fig. 2 shows the quality of the corresponding fit based on Eq. (1).

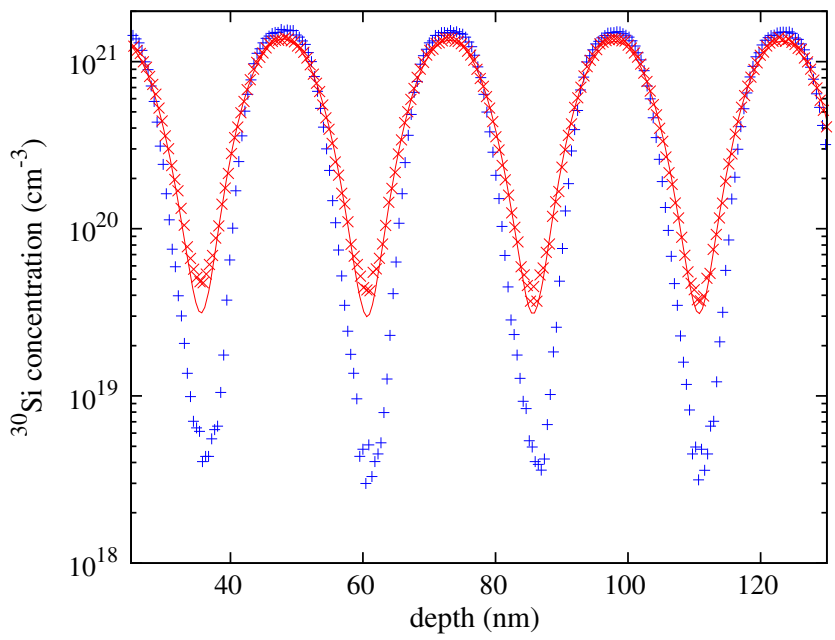

FIG. 2. ${ }^{30} \mathrm{Si}$ concentration-depth profiles measured with SIMS after annealing of sample type \#1 at $550^{\circ} \mathrm{C}$ for $5 \mathrm{~h}(\times)$ in comparison to the profile $(+)$ after relaxation $\left(\mathrm{T}=520^{\circ} \mathrm{C}\right.$, $\mathrm{t}=15 \mathrm{~min})$. The red solid line is the best fit to the experimental diffusion profile that is based on numerical solutions of Eq. (1) considering the self-atom profile measured after relaxation as initial profile. 
The self-diffusion coefficients determined from the analyses of all samples from set \#1 and \#2 are listed in Table S1 of the Supplemental Material [16]. Diffusion annealing at fixed temperatures for various times reveals that $D_{\mathrm{Si}}^{\mathrm{SD}}$ deviates at maximum by about $20 \%$ (see Table $\mathrm{S} 1$ of Supplemental Material [16]). This deviation is within experimental accuracy and demonstrates that self-diffusion in the considered temperature-time frame does not change with time. This supports a relaxed metastable $a$-Si structure whose dynamics on the atomic scale becomes visible by the interfacial broadening of the isotope structure.

Figure 3 shows the temperature dependence of the selfdiffusion coefficients in comparison to results reported by Strauß et al. [3] and Noah et al. [21]. The temperature dependence is best described by an Arrhenius equation $D_{\mathrm{Si}}^{\mathrm{SD}}(T)=D_{0} \exp \left(-Q / k_{B} T\right)$ with an activation enthalpy $Q=(2.70 \pm 0.11) \mathrm{eV}$ and preexponential factor $D_{0}=\left(5.5_{-3.7}^{+11.1}\right) \times 10^{-2} \mathrm{~cm}^{2} \mathrm{~s}^{-1}$. The self-diffusion data reported in this work are at variance to the results of Strauß et al. [3] but confirm the data given by Noah et al. [21]. Noah et al. determined the self-diffusion coefficients in $a$-Si from the interdiffusion of $a-\mathrm{Si} / a-\mathrm{Si}_{0.52} \mathrm{Ge}_{0.48}$ multilayer structure that was prepared by evaporation of $\mathrm{Si}$ and $\mathrm{Ge}$ in ultra high vacuum on a (100)-oriented Si substrate covered with $\mathrm{Si}_{3} \mathrm{~N}_{4}$. The deposition on top of the silicon nitride layer avoids direct contact of the amorphous $\mathrm{Si} / \mathrm{SiGe}$ multilayer structure with the crystalline substrate and thus impedes SPER during diffusion annealing. Strauß et al. prepared a multilayer structure with ten bilayers of

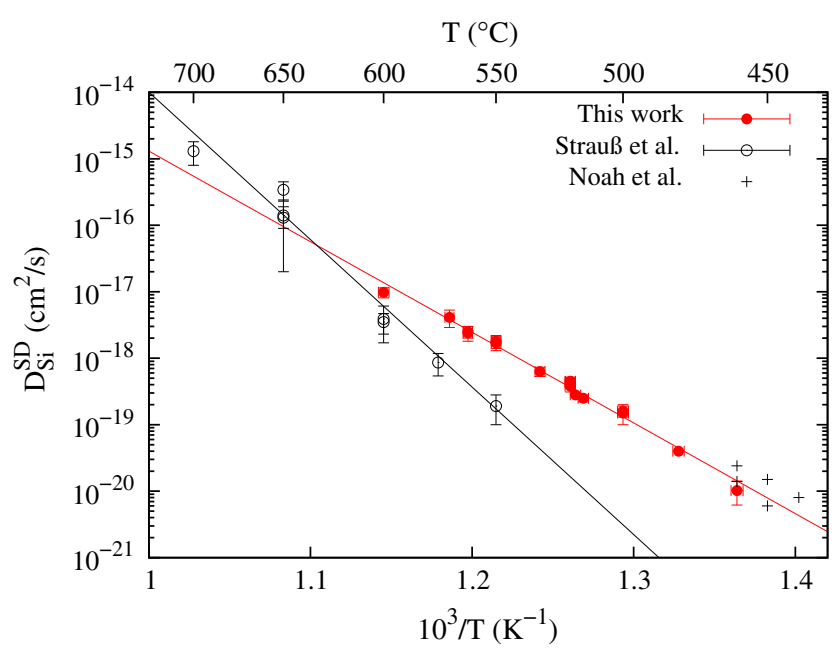

FIG. 3. Self-diffusion coefficient $D_{\mathrm{Si}}^{\mathrm{SD}}$ in amorphous $\mathrm{Si}$ versus the inverse temperature $10^{3} / T$. The temperature dependences of self-diffusion deduced from the data of this work (solid circle) and of Strauß et al. [3] (circle) are described by an Arrhenius equation with $Q=(2.70 \pm 0.11) \mathrm{eV}, D_{0}=\left(5.5_{-3.7}^{+11.1}\right) \times 10^{-2} \mathrm{~cm}^{2} \mathrm{~s}^{-1}$ (red line) and $Q=(4.4 \pm 0.3) \mathrm{eV}, D_{0}=\left(1.5_{-1.5}^{+45.9}\right) \times 10^{8} \mathrm{~cm}^{2} / \mathrm{s}$ (black line), respectively. It is obvious that the data of this work deviate from those of Strauß et al. but agree with the data (plus) of Noah et al. [21].
${ }^{29} \mathrm{Si} /{ }^{\text {nat }} \mathrm{Si}$ by sputter deposition on a (100)-oriented $\mathrm{Si}$ substrate. As outlined above, it is likely that the amorphous structure prepared by Strauß et al. is stabilized by a high level of carbon impurities $(\leq 5 \%)$. Accordingly, the results reported by $\mathrm{Strau} ß$ for self-diffusion in $a$-Si remain questionable.

In accord with the results of Noah et al. [21], we could not detect any significant interfacial broadening due to structural relaxation. This confirms that the relaxation is a local atomic reordering process [10]. The activation enthalpy $Q=(2.70 \pm 0.11) \mathrm{eV}$ determined in this work for self-diffusion in $a$-Si equals the activation enthalpy for the SPER rate $(Q=2.75 \mathrm{eV}$ [7]), the diffusion activation enthalpy of hydrogen $(\mathrm{H})$ in $a$-Si for low $\mathrm{H}$ concentrations $(Q=2.7 \mathrm{eV}$ [22]), and the diffusion of coordination or bond defects $[Q=(2.6 \pm 0.4) \mathrm{eV}]$, i.e., dangling and floating bonds in the case of three- and fivefold coordinated $\mathrm{Si}$ atoms, respectively. The latter value was deduced from the model of boron diffusion in $a$-Si [23]. Hydrogen is known to saturate dangling bonds in $a$-Si [24] while SPER is considered to be mediated by local bond rearrangements at the crystalline-amorphous interface [7]. The overall similarity in the $\mathrm{Q}$ values gives strong evidence that bond switching or bond rearrangement mechanisms governs also the self-diffusion process in $a$-Si.

Roorda et al. [10] report a concentration of bond defects of about $1 \%$ in relaxed $a-\mathrm{Si}$. This concentration of defects is several orders of magnitude higher than the equilibrium concentrations of self-interstitials and vacancies [25] that mediate self-diffusion in crystalline $\mathrm{Si}(c$-Si) [2]. On the other hand, self-diffusion in $a$-Si at e.g. $550^{\circ} \mathrm{C}$ is about nine orders of magnitude faster than results reported by Südkamp et al. [2] for self-diffusion in $c$-Si extrapolated to low temperatures. This strong disparity may lead to the conclusion that the relation between the magnitude of selfdiffusion in c- and $a$-Si is determined by different defect concentrations. However, theoretical investigations showed that bond exchange or switching in bond-defect-free, i.e., fully fourfold coordinated $a$-Si, can also lead to atomic migration (see below).

Strauß et al. [3] suggest that the high value of $Q=$ $(4.4 \pm 0.3) \mathrm{eV}$ deduced from their self-diffusion study is composed of a defect formation and migration part. The nature of the defect mediating self-diffusion is motivated by the high diffusion activation entropy $S^{\mathrm{SD}}=24 k_{B}$ deduced from the preexponential factor of the temperature dependence of self-diffusion [3]. This high value led Strauß et al. to suppose the presence of extended defects that incorporate several atoms. The concept of extended defects was first introduced by Seeger and Chik [26] to explain the high preexponential factor of self-diffusion in $c$-Si. Recently this concept was renewed by Cowern et al. [27] to explain the diffusion of B in c-Ge and suggested to be applicable for $c$ $\mathrm{Si}$ and many other crystalline solids. The extended defect is considered to be similar to an amorphous pocket [27] in a 
crystalline structure. Seemingly, the work of Strauß et al. supports an extended defect model. However, the results reported in this work demonstrate that the experiments on self-diffusion in $a$-Si performed by Strauß et al. [3] are erroneous and therewith not capable to draw any conclusion about the presence of extended defects. Considering the preexponential factor $D_{0}=\left(5.5_{-3.7}^{+11.1}\right) \times 10^{-2} \mathrm{~cm}^{2} \mathrm{~s}^{-1}$ for self-diffusion in $a$-Si obtained in this work and the equation $D_{0}=\nu_{0} a_{0}^{2} \exp \left(S^{\mathrm{SD}} / k_{B}\right)$ with the jump frequency $\nu_{0} \approx 10^{13} \mathrm{~s}^{-1}$ on the order of the Debye frequency and the interatomic distance $a_{0}=0.235 \mathrm{~nm}$, a diffusion activation entropy of $S^{\mathrm{SD}} \approx(2.3 \pm 1.1) k_{B}$ is obtained. This value and the activation enthalpy $Q=(2.70 \pm 0.11) \mathrm{eV}$ are substantially lower than the corresponding data reported by Strauß et al. [3,4]. Therewith, our results do not support the concept of extended defects mediating self-diffusion in $a$ $\mathrm{Si}$. Instead, the close similarity between the activation enthalpy of self-diffusion and the activation enthalpy of other processes in $a$-Si rather support local bond rearrangements as dominating mechanisms.

Evidences on the mechanisms of atomic migration in $a-\mathrm{Si}$ can be also obtained from theoretical calculations. However, the few atomistic simulations on self-diffusion in $a-\mathrm{Si}$ hitherto performed led to very different results. Using the original Stillinger-Weber interatomic potential Sastry et al. [28] obtained a value of $6.4 \times 10^{-8} \mathrm{~cm}^{2} \mathrm{~s}^{-1}$ for the selfdiffusion coefficient at $1060 \mathrm{~K}$. On the other hand, the extrapolation of our experimental data to $1060 \mathrm{~K}$ yields $8.0 \times 10^{-15} \mathrm{~cm}^{2} \mathrm{~s}^{-1}$. A high self-diffusion coefficient was also found by tight-binding molecular dynamics (MD) simulations of Santos et al. [29]. These authors considered both defect-free $a$-Si prepared by the WWW algorithm [30] and a sample prepared by quenching from the liquid phase with $20 \%$ fivefold-coordinated atoms. In the first and the second case, the obtained self-diffusion coefficients were $D_{\mathrm{Si}}^{\mathrm{SD}}(T)=4.2 \times 10^{-4} \exp \left(-0.95 \mathrm{eV} / k_{B} T\right) \mathrm{cm}^{2} \mathrm{~s}^{-1}$ and $D_{\mathrm{Si}}^{\mathrm{SD}}(T)=2.8 \times 10^{-4} \exp \left(-0.86 \mathrm{eV} / k_{B} T\right) \mathrm{cm}^{2} \mathrm{~s}^{-1}\left(1.3 \times 10^{-8}\right.$ and $2.3 \times 10^{-8} \mathrm{~cm}^{2} \mathrm{~s}^{-1}$ at $1060 \mathrm{~K}$ ), respectively. The results of Ref. [29] demonstrate that the diffusion activation energy should not significantly depend on whether coordination defects are present or not. Santos et al. [29] identified five types of diffusion mechanisms, such as bond breaking and bond switching. Song et al. [31] used a modified StillingerWeber-type potential and the nudged elastic band method, in combination with the activation-relaxation technique, to determine the energy barriers between the plethora of states possible in a defect-free (fourfold coordinated) $a$ $\mathrm{Si}$ structure. They obtained an average barrier height of about $3 \mathrm{eV}$. This value is not very different from our experimental value for the activation enthalpy. Song et al. [31] found that the WWW bond switching, or exchange [30], is the most important mechanism for migration events in their $a$-Si material. The results of Song et al. [31] reveal a preexponential factor of about $10^{-2} \mathrm{~cm}^{2} \mathrm{~s}^{-1}$ and suggest a self-diffusion coefficient of $a$-Si of about $5 \times 10^{-17} \mathrm{~cm}^{2} \mathrm{~s}^{-1}$ at $1060 \mathrm{~K}$. The above overview reveals strong differences between the results obtained so far in theoretical investigations, although the relevant mechanisms discussed are rather similar. The experimental data provided in this work form a solid basis and a firm benchmark for future theoretical work.

In summary, we have studied self-diffusion in amorphous silicon isotope multilayer structures at temperatures between $460-600^{\circ} \mathrm{C}$. The temperature dependence of selfdiffusion follows an Arrhenius equation with an activation enthalpy of $Q=(2.70 \pm 0.11) \mathrm{eV}$ and preexponential factor $D_{0}=\left(5.5_{-3.7}^{+11.1}\right) \times 10^{-2} \mathrm{~cm}^{2} \mathrm{~s}^{-1}$. The activation enthalpy equals the activation enthalpy of solid-phase epitaxial recrystallization [6], of the migration of dangling and floating bonds [23], and of the diffusion of hydrogen [22] in amorphous silicon. The remarkable agreement shows that the mechanisms mediating these processes must have a common origin. Based on present studies, it is argued that self-diffusion in amorphous silicon is mediated by local bond rearrangements. This questions recent results on self-diffusion reported by Strauß et al. [3] and, thus, also their conclusion on the presence of extended defects in amorphous and crystalline $\mathrm{Si}$.

The authors thank Dr. H. Bettin and Dr. P. Becker from PTB (Braunschweig) for the ${ }^{28} \mathrm{Si}$ enriched material that was prepared from Avo28 crystal produced by the International Avogadro Coordination (IAC) Project in cooperation among the Bureau International des Poids et Mesures, the Istituto Nazionale du Ricerca Metrologica (Italy), the Institute of Reference Materials and Measurements (EU), the National Measurement Institute of Australia (Australia), the National Metrology Institute of Japan (Japan), the National Physical Laboratory (UK), and the Physikalisch-Technische Bundesanstalt (Germany). We appreciate the help of S. Hilke and F. Emeis in the analyses of our samples with TEM and EBSD, respectively. Support by the Ion Beam Center at Helmholtz-Zentrum DresdenRossendorf and the DFG under Grant No. BO 3140/4-1 is gratefully acknowledged.

*m.posselt@hzdr.de

bracht@wwu.de

[1] H. Bracht, E. E. Haller, and R. Clark-Phelps, Phys. Rev. Lett. 81, 393 (1998).

[2] T. Südkamp and H. Bracht, Phys. Rev. B 94, 125208 (2016).

[3] F. Strauß, L. Dörrer, T. Geue, J. Stahn, A. Koutsioubas, S. Mattauch, and H. Schmidt, Phys. Rev. Lett. 116, 025901 (2016); [116, 089903(E) (2016)].

[4] F. Strauß, B. Jerliu, T. Geue, J. Stahn, and H. Schmidt, J. Appl. Phys. 119, 175102 (2016).

[5] I. Mizushima, Y. Mitani, K. Miyano, and S. Kambayashi, Extended Abstracts of the 1999 International Conference on Solid State Devices and Materials, Tokyo (The Japanese Society of Applied Physics, Tokyo, 1999), pp. 36-37. 
[6] G. L. Olson and J. A. Roth, Mater. Sci. Rep. 3, 1 (1988).

[7] B. C. Johnson, J. C. McCallum, and M. J. Aziz, in Handbook of Crystal Growth, editor T. F. Kuech (North-Holland, Boston, 2015), 2nd ed., p. 317.

[8] J. W. Strane, S. R. Lee, H. J. Stein, S. T. Picraux, J. K. Watanabe, and J. W. Mayer, J. Appl. Phys. 79, 637 (1996).

[9] E. F. Kennedy, L. Csepregi, J. W. Mayer, and T. W. Sigmon, J. Appl. Phys. 48, 4241 (1977).

[10] S. Roorda, J. S. Custer, W. C. Sinke, J. M. Poate, D. C. Jacobson, A. Polman, and F. Spaepen, Nucl. Instrum. Methods Phys. Res., Sect. B 59-60, 344 (1991).

[11] S. Roorda and Y. Lavigueur, Philos. Mag. 90, 3787 (2010).

[12] J.-F. Mercure, R. Karmouch, Y. Anahory, S. Roorda, and F. Schiettekatte, Phys. Rev. B 71, 134205 (2005).

[13] E. P. Donovan, F. Spaepen, D. Turnbull, J. M. Poate, and D. C. Jacobson, J. Appl. Phys. 57, 1795 (1985).

[14] E. P. Donovan, F. Spaepen, J. M. Poate, and D. C. Jacobson, Appl. Phys. Lett. 55, 1516 (1989).

[15] S. Roorda, S. Doorn, W. C. Sinke, P. M. L. O. Scholte, and E. van Loenen, Phys. Rev. Lett. 62, 1880 (1989).

[16] See Supplemental Material at http://link.aps.org/ supplemental/10.1103/PhysRevLett.120.225902 for details on the experiments and analyses of self-diffusion in amorphous silicon and the Table summarizing the self-diffusion coefficients determined in this work. The Supplemental Material includes Refs. [2,17-20].

[17] J. F. Ziegler, M. D. Ziegler, and J.P. Biersack, Nucl. Instrum. Methods Phys. Res., Sect. B 268, 1818 (2010).
[18] J. F. Ziegler, J. P. Biersack, and U. Littmark, in The Stopping and Range of Ions in Solids (Pergamon, New York, 1985).

[19] W. Wisniewski, S. Saager, A. Böbenroth, and Chr. Rüssel, Ultramicroscopy 173, 1 (2017).

[20] M. Radek, H. Bracht, B. Liedke, R. Böttger, and M. Posselt, J. Appl. Phys. 120, 185701 (2016).

[21] M. A. Noah, D. Flötotto, Z. Wang, and E. J. Mittemeijer, J. Appl. Phys. 117, 165306 (2015).

[22] J. A. Roth, G. L. Olson, D. C. Jacobson, and J. M. Poate, Mater. Res. Soc. Symp. Proc. 297, 291 (1993).

[23] S. Mirabella, D. De Salvador, E. Bruno, E. Napolitani, E. F. Pecora, S. Boninelli, and F. Priolo, Phys. Rev. Lett. 100, 155901 (2008).

[24] R. A. Street, Physica B (Amsterdam) 170, 69 (1991).

[25] H. Bracht, N. A. Stolwijk, and H. Mehrer, Phys. Rev. B 52, 16542 (1995).

[26] A. Seeger and K. P. Chik, Phys. Status Solidi 29, 455 (1968).

[27] N. E. B. Cowern, S. Simdyankin, C. Ahn, N. S. Bennett, J. P. Goss, J.-M. Hartmann, A. Pakfar, S. Hamm, J. Valentin, E. Napolitani, D. De Salvador, E. Bruno, and S. Mirabella, Phys. Rev. Lett. 110, 155501 (2013).

[28] S. Sastry and C. A. Angell, Nat. Mater. 2, 739 (2003).

[29] I. Santos, L. A. Marqués, L. Pelaz, and L. Colombo, Phys. Rev. B 83, 153201 (2011).

[30] F. Wooten, K. Winer, and D. Weaire, Phys. Rev. Lett. 54, 1392 (1985).

[31] Y. Song, R. Malek, and N. Mousseau, Phys. Rev. B 62, 15680 (2000). 